\title{
Massive carcinoma of the cornea in an immunocompetent patient
}

\author{
Irene Pecorella ${ }^{1}$, Pierluigi Grenga ${ }^{2}$, Enzo Maria Vingolo ${ }^{2}$ \\ ${ }^{1}$ Department of Experimental Medicine, University of Rome “Sapienza” Viale Regina Elena, Rome, Italy; \\ *Corresponding Author: irene.pecorella@uniroma1.it \\ ${ }^{2}$ Department of Ophthalmologic Science, University of Rome “Sapienza” Viale Regina Elena, Rome, Italy; \\ pigig@tiscalinet.it; e.vingolo@rdn.it
}

Received 11 February 2011; revised 7 March 2011; accepted 29 March 2011.

\begin{abstract}
A case of massive well-differentiated, superficially invasive, squamous cell carcinoma of the cornea is described. This patient did not have any common identifiable risk factors in the development of SCC of the ocular surface, such as excessive solar exposure, HPV infection, immunocompromised state, or chronic ocular irritation. A perforating injury in the cornea of the same eye had occurred 11 years before. The possible role of trauma in causing subsequent neoplastic development is discussed, as well as the immunohistochemical results for p53 and p63.
\end{abstract}

Keywords: Squamous Cell Carcinoma; Cornea; HPV; Trauma; Solar Elastosis

\section{INTRODUCTION}

Squamous cell carcinoma (SCC) is the most common primary malignancy of the ocular surface, making up $14 \%$ of all primary ocular and orbital tumors. It predominantly affects male patients with an average age of 56 years (range: 4 to $91 \mathrm{yrs}$ ). SCC most commonly occurs in the limbus (87.8\%), where the corneal epithelial stem cells are located [1], and in the interpalpebral space, where the ocular tissues are mostly exposed to ultraviolet irradiation. From this location, the tumour may spread onto the bulbar conjunctiva and/or the cornea. SCC of the cornea without involvement of the conjunctiva and limbus is a very rare condition. The corneal intrastromal infiltration is clinically described as corneal cystic gelatinous lesion [1]. Intraocular invasion is often heralded by the onset of low-grade inflammation and secondary glaucoma. Metastasis is uncommon and, when present, usually occurs in the preauricular, submandibular or cervical lymph nodes, the parotid gland, the lungs or the bones.

Its origin is debated and some investigators believe that the corneal epithelium may undergo dysplastic and cancerous changes, whereas the limbal transition zone/ stem cell theory has been proposed for the development of corneal intraepithelial neoplasia by Lee and Hirst [1]. Recently, Chikama et al demonstrated in transgenic mice lines that upregulation of FGF-7 signalling pathway(s) may be common in tumorigenesis derived from limbal stem cells that undergo oncogenic transformation by insults, such as long-term ultraviolet B (UVB) exposure, infection of human papilloma virus (HPV) and human immune deficiency virus (HIV), and cigarette smoking, etc. [2].

We present herein a case of massive SCC of the limbus and cornea in an immunocompetent patient and discuss the possible etiological role of a preceding traumatic event to the same eye in causing the occurrence of the tumor.

\section{CLINICAL HISTORY}

A 67-years-old male patient presented with a painful eye and a massive, protuberant lesion covering the nasal half of the left cornea (Figure 1). The lesion had initially appeared in the nasal limbal area and had been slowly enlarging to its present size for approximately 18 months.

Eleven years before a perforating injury in the left cornea of the same eye, had caused progression to bulbar phthisis. His work history did not involve exposure to radiation, chemicals, or the sun. The patient was otherwise in good health.

Ophthalmological examination showed a white, fleshy papillomatous mass, hanging from the surface of the left cornea. The neighbouring conjunctiva was markedly hyperaemic. A clinical diagnosis of squamous cell carcinoma of the ocular surface was made. Visual acuity was of hand motion near to face. Digital tonometry revealed 


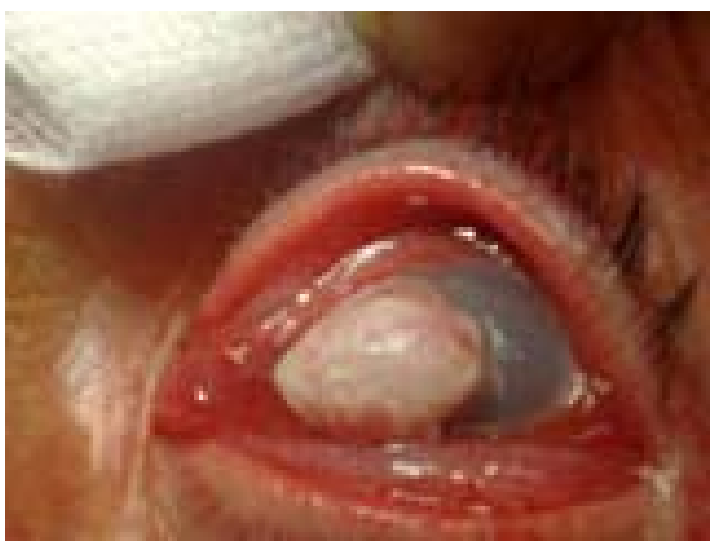

Figure 1. Clinical picture of the large exophitic neoplasm covering the nasal half of the left cornea

a hard consistency of the affected painful eye. The anterior chamber structure could not be evaluated due to the lack of corneal transparency. Ultrasound B-scans did not reveal any posterior segment neoplastic involvement. Regional lymph nodes were not palpable.

Visual acuity in the contralateral eye was 10/10 and no abnormalities were observed at slit-lamp biomicroscopic examination. Laboratory examination, including complete blood count and HIV and HPV tests were normal and a clinical work-up demonstrated no metastatic disease. The blind eye was eviscerated for cosmetical reasons and the cornea and the ocular content were sent for histological examination.

The postoperative course was uneventful and the patient is free of local or distant recurrence 14 months postoperatively.

\section{MATERIAL, METHODS AND RESULTS}

The tumoral mass and the ocular content were formalin fixed and paraffin embedded. The anterior portion of the eviscerated material consisted of a corneo-scleral button measuring 1.3 in diameter with an overlying exophytic mass of $1.5 \times 1 \times 0.4$ and circumferential peripheral retrocorneal uveal synechiae

Microscopically, the lesion was consistent with a wellcircumscribed, well-differentiated squamous cell carcinoma (SCC), forming keratin pearls. Invasion of the superficial corneal stroma was observed $(<1 \mathrm{~mm})$ (Figure 2). Descemet's membrane was focally interrupted and plicated and the endothelial layer attenuated. The excision margin was clear. At the periphery of the corneal button, a fragment of iris incarcerated in the deep stroma was observed. Remnants of atrophic iris formed anterior synechiae and the pupil was obliterated by a fibro-inflammatory membrane.

Examination of the ocular content revealed a gliotic retina with overlying fibrovascular epiretinal membrane.

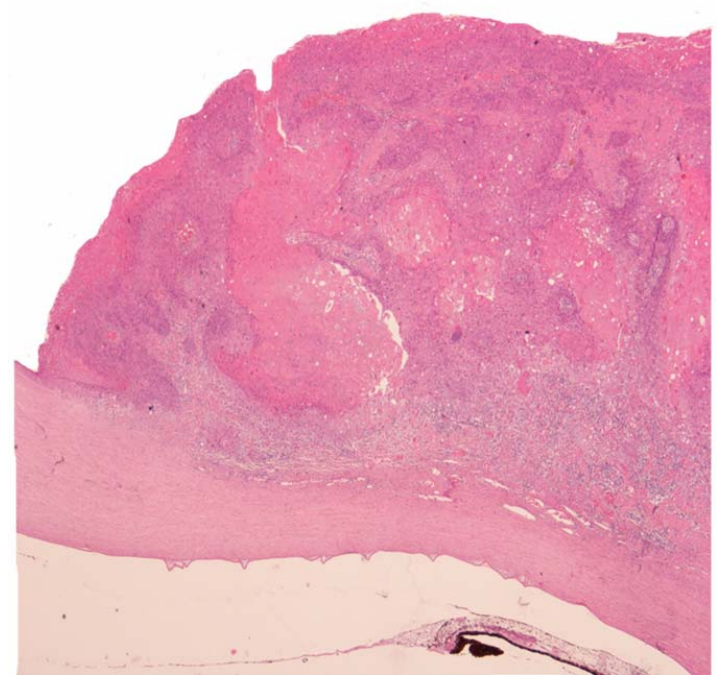

Figure 2. Histological appearance of the corneal mass. A post-inflammatory fibrous pupillary membrane is also visible (Haematoxylin-eosin $25 \times$ ).

Foci of chronic choroiditis and areas of subretinal osseous metaplasia were also apparent.

Sections of the tumoral mass were stained immunohistochemically for p53, p63 and the proliferation marker Ki67.

The p53 gene acquires mutations during the development of many human malignancies, including SCC of the ocular surface [3]. Mutations of the p53 gene and overexpression of p53 protein are closely related events. p63 is a homologue of the tumour suppressor gene p53, but acts as an oncogene and is overexpressed in squamous cell dysplasias or neoplasias.

Results showed a positive reaction for p53 in most of the nuclei of the neoplastic cells. The positive cells were mainly located in the deep infiltrating tongues and in the basal layers of the superficial tumoral elements (Figure 3). Positive non tumoral epithelial cells were observed at the limbus, as well.

A widespread strong nuclear staining in malignant cells was observed using antibodies for Ki67 or p63. In the adjacent non-affected cornea, the positive staining for $\mathrm{p} 63$ was confined to the basal cell layer.

\section{DISCUSSION}

The corneal SCC is a slow growing tumour that remains localized. Massive epithelial carcinomas involving primarily the cornea are unusual, accounting for $0,76 \%$ of cases in one large series of conjunctival SCC [4]. The tumour size in this case was relatively large. Large-sized tumours may be related to well differentiation of the neoplasm, and to slow progressive growth. Alternatively, the difficulty to infiltrate dense structures 


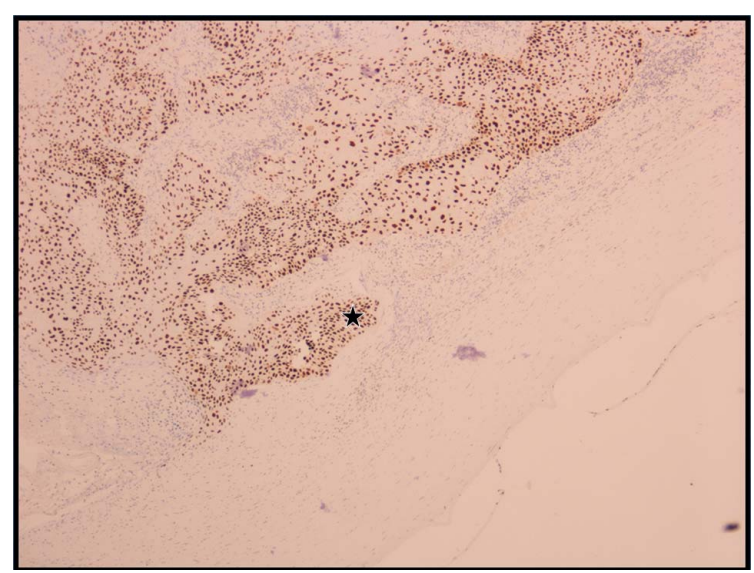

Figure 3. Immunohistochemical detection of p53 in the nuclei (brown color) of the malignant cells. Diffuse nuclear positivity in the infiltrating tongue of cancer cells invading the anterior corneal stroma (asterisk) (Streptavidinbiotin- DAB staining $100 \times$ ).

like the cornea and the sclera may favour a superficial growth for a long time and an exophytic pattern in longstanding lesions.

Our patient did not have any of the several identifiable risk factors in the development of SCC of the ocular surface, such as excessive solar exposure, HPV infection, immunocompromised state such as in HIV, a history of smoking, or chronic irritation from dust, wind petroleum exposure.

Mechanical trauma to the ocular surface, including thermal burns, is occasionally mentioned among the risk factors for SCC of the eye. Two of the 17 cases of conjunctival SCC listed by Auw-Haedrich and coll. had suffered from ocular injury many years before the occurrence of the neoplasm [5]. Three cases were reported in an orbital socket after long wearing of ocular prostheses [6]. Scars can promote malignant growth of tumour cells. Thick, dense scars are the type that usually gives rise to squamous cell carcinoma of the skin and the cicatrix may be caused by any traumatic event. Surgical incisions have also been claimed to cause skin cancer. Trauma is considered not to act as initiator, but to act as promoter of carcinogenesis. There are two theories as for the induction of scar cancer. One is that the cells composing scar tissues convert themselves directly into tumorigenic ones; the other is that scar tissues secrete soluble factors that stimulate carcinogenic conversion or proliferation of tumour cells pre-existing around the scar [7]. Whether fibrosis is accompanied with tumour or not depends on the types of collagen it produces. Tumourassociated fibrosis is characterized by increased collagen Type III content [8]. According to Martins-Green and coll., during the inflammatory response caused by wounding, a plethora of cytokines are released that cause blood vessel leakage and stimulate cell division which are key elements in the process leading to possible viral oncogene integration and activation and then to tumour development. In their experimental work, wounding at 4 or 5 days after inoculation of Rous sarcoma virus resulted in $100 \%$ of the chickens developing wound tumours [7]. Their findings casts a new light on the possible role of viruses in tumour development and, possibly, on preexisting HPV ocular infection and post-traumatic neoplasm.

We believe that the previous injury to the eye may have played a role in the development of the corneal neoplasia in this patient. However, we cannot dismiss the possibility that an actinic damage not related to outdoor working might have contributed to the development of the corneo/conjunctival neoplasia, as pointed out by the diffuse immunopositivity to p53. Normally, p53 is undetectable immunohistochemically and negative in normal human conjunctiva. Mutations of p53 are characteristic of solar damage and are detected in the majority of SCC of the skin and in around 50\% of the conjunctival SCC. Also Toth and coll. recently reported p53 gene overexpression in $78 \%$ of conjunctival SCC and found no relationship with HPV type 16 or 18 infection within the tumour tissue [4]. In a bilateral SCC of the conjunctiva, p53 positivity was found in only one of the eyes [9], perhaps indicating that the presence of mutant p53 is rather a marker for tumour aggressiveness. Interestingly, also the limbal cells overexpressed this tumour marker in our specimen. Its detection may be related to its mutation or, alternatively to its angiogenic activity in an eye with a previous penetrating injury and ocular surface remodelling.

Our immunohistochemical results also confirm the previously reported p63 expression in the neoplastic cells of the conjunctival SCC and its distribution in all layers of this invasive carcinoma [6]. Its detection may prove useful in the differential diagnosis between reactive and malignant neoplastic lesions of the conjunctiva.

\section{REFERENCES}

[1] Lee, G.A. and Hirst, L.W. (1997) Retrospective study of ocular surface squamous neoplasia. Aust NZJ Ophthalmol, 25, 269-276. doi:10.1111/j.1442-9071.1997.tb01514.x

[2] Chikama, T., Liu, C.Y., Meij, J.T.A., Hayashi, Y., Wang, I.J., Yang, L., Nishida, T. and Kao, W.W.Y. (2008) Excess FGF-7 in corneal epithelium causes corneal intraepithelial neoplasia in young mice and epithelium hyperplasia in adult mice. Am J Pathol, 172, 638-649. doi:10.2353/ajpath.2008.070897

[3] Toth, J., Karcioglu, Z.A., Moshfeghi, A.A., Issa, T.M., Al-Ma'ani, J.R. and Patel, K.V. (2000) Correlation between HPV positivity and p53 gene mutation in conjunc- 
tival squamous cell carcinoma. Cornea, 19, 159-162. doi:10.1097/00003226-200003000-00007

[4] McKelvie, P.A., Daniell, M., McNab, A., Loughnan, M. and Santamaria, J.D. (2002) Squamous cell carcinoma of the conjunctiva: a series of 26 cases. Br J Ophthalmol, 86, 168-173. doi:10.1136/bjo.86.2.168

[5] Auw-Haedrich, C., Sundmacher, R., Freudenberg, N., Spelsberg, H., Feltgen, N., Maier, P. and Reinhard, T. (2006) Expression of p63 in conjunctival intraepithelial neoplasia and squamous cell carcinoma. Graefe's Arch Clin Exp Ophthalmol, 244, 96-103. doi:10.1007/s00417-005-0025-4

[6] Pe'er, J. (2005) Ocular surface squamous neoplasia. Oph- thalmol Clin N Am, 18, 1-13.

[7] Martins-Green, M., Boudreau, N. and Bissell, M.J. (1994) Inflammation is responsible for the development of wound-induced tumors in chickens infected with Rous sarcoma virus. Cancer Res, 54, 4334-4341.

[8] van den Hooff, A. (1988) Stromal involvement in malignant growth. Adv Cancer Res, 50, 159-196. doi:10.1016/S0065-230X(08)60437-6

[9] Usui, Y., Waring, G.O., See, R.F. and Rao, N.A. (2004) Bilateral ocular surface squamous cell neoplasia: a clinicopathological case report. Br J Ophthalmol, 88, 595-596. doi:10.1136/bjo.2003.029710 